Gil Bazanini iD

gil.bazanini@udesc.br

Universidade do Estado de

Santa Catarina (UDESC)

Ricardo Kirchhof Unfer ricardo.unfer@udesc.br Universidade do Estado de Santa Catarina (UDESC)

Nicodemus Neto da Costa Lima

nicodemus.lima@udesc.br Universidade do Estado de Santa Catarina (UDESC)

\title{
Crescimento e colapso de bolhas - uma comparação com o modelo de universo finito
}

\section{Bubbles growth and collapse - a comparison with the finite universe model}

\section{Crecimiento y colapso de burbujas - comparación con el modelo de universo finito}

\section{Croissance et l'effondrement des bulles - Comparaison avec le modèle de l'univers fini}

\section{Resumo}

No presente trabalho são estudadas numericamente oscilações de bolhas excitadas por um campo externo variável de pressão. O modelo apresentado é mais completo que outros existentes por considerar a presença de ar mais vapor como gases reais dentro da bolha e as propriedades físicas dos fluidos participantes do processo, tais como viscosidade, densidade e tensão superficial. Finalmente, o modelo utilizado é comparado ao modelo de universo finito fechado (atualmente em expansão) e eterno visando obter eventuais conclusões sobre um possível futuro, resultando em um universo finito eterno, concordando com o modelo proposto pelo cientista brasileiro Mário Novello.

Palavras-chave: Bolhas. Oscilações. Universo.

\begin{abstract}
Cavities oscillations driven by an external pressure field are studied using numerical simulation in the present work.

The model presented here is more complete than the existing ones, since its equation takes into account the presence of air plus vapor in the cavity, as a mixture of real gases. It also considers the physical properties of the fluids involved such as viscosity and vapor pressure. Finally, the model is used in comparison with the finite spherical universe model, resulting in an eternal finite universe, in agreement with the model proposed by the Brazilian scientist Mário Novello.
\end{abstract}

Keywords: Bubble. Oscillation. Universe.

\section{Resumen}

En este trabajo son estudiadas numéricamente oscilaciones de burbujas excitadas por un campo externo variable de presión. El modelo presentado es más completo que otros existentes por considerar la presencia de aire más vapor con gases reales dentro de la burbuja y las propiedades físicas de los fluidos participantes del proceso tales como viscosidad, densidad, tensión superficial. Finalmente, el modelo utilizado es comparado al modelo de universo finito cerrado (actualmente en expansión) y eterno, buscando obtener eventuales conclusiones sobre un posible futuro destinado al mismo, resultando en un universo finito eterno, de acuerdo (sino con base) con el modelo propuesto por el científico brasileño Mário Novello.

Palabras-clave: Burbujas. Oscilaciones. Universo. 


\section{Résumé}

Dans le présent travail, on étude des oscillations de bulles excitées par un champ de pression externe variable. Le modèle présenté est plus complet que d'autres, parce qu'il considère la présence d'air plus de vapeur en tant que gaz réels à l'intérieur de la bulle. Ce modèle considère aussi des propriétés physiques des fluides qui participent au processus telles que la viscosité, la densité, la tension superficielle. Enfin, le modèle utilisé est comparé au modèle de l'univers fermé (actuellement en expansion) et éternel, ce qui vise à tirer des conclusions sur un éventuel futur qui lui est destiné, en résultant à un univers fini éternel, en accord avec le modèle proposé par le scientifique brésilien Mário Novello.

Mots clés: Bulles. Oscillations. Univers.

\section{Introdução}

Desde os primórdios o ser humano tem atuado como contemplador (e, posteriormente, como observador) do céu, diurno e noturno, associando, inicialmente, a posição dos astros às estações do ano e, inevitavelmente, objetivando sua subsistência (também associada fatores de cunho holísticos), baseada no plantio, irrigação e colheitas. Posteriormente, isto o levou a questionamentos sobre a configuração do universo em que vivemos. As observações, que continuam nos tempos atuais, embaladas pelo advento dos telescópios e satélites, foram aperfeiçoadas com o avanço inexorável do desenvolvimento tecnológico.

As modernas concepções científicas da cosmologia (que estuda a origem e evolução do universo) são evoluções paradigmáticas naturais profundamente transformadas pela centralização em torno do racionalismo, bem como de uma observação mais rigorosa da natureza. A boa aceitação do modelo cosmológico do "Big Bang", associada a observações rigorosas suportadas pela tecnologia contemporânea, levou à conclusão absoluta de um universo (atualmente) em plena expansão, já previstas nas equações da relatividade geral propostas pelo físico alemão Albert Einstein quando solucionadas por Friedmann e Lemâitre.

A questão imediatamente subsequente, e ainda por ser solucionada, refere-se à geometria do universo, considerado homogêneo (mesmas propriedades em qualquer local do espaço) e isotrópico (mesmas propriedades em qualquer direção do espaço) (EINSTEIN, 1953), dentro de três possibilidades, a saber: esférico fechado, plano ou hiperbólico aberto (HAWKING, 2016).

Foi acrescentada na equação da relatividade geral uma constante (k) que, dependendo do valor assumido, considera um determinado tipo de geometria para o universo (BERMAN; GOMIDE, 1987). Em coordenadas esféricas, que é o sistema de referências apropriado ao estudo desse modelo de universo, resulta em:

$$
d s^{2}=A(r, t) d t^{2}+B(r, t) d r^{2}+c(r, t)\left(d \theta^{2}+\operatorname{sen}^{2} \theta d \phi^{2}\right)
$$

Onde $s$ é o espaço $\varnothing, \theta$ e $r$ são as direções referentes ao sistema de coordenadas adotado, e $t$ é o tempo. Comparando a Eq. (1) com a Eq. (2) abaixo, de Robertson-Walker (RIVELLES, 2009):

$$
d s^{2}=c^{2} d t^{2}-R^{2}(t)\left[\frac{d r^{2}}{1-k r^{2}}+r^{2}\left(d \theta^{2}+\operatorname{sen}^{2} \theta d \phi^{2}\right)\right]
$$

Onde $c$ é a velocidade da luz no vácuo (aproximadamente 300.000 kilômetros por segundo), $r(t)$ é o raio e $k$ é a constante associada à geometria do universo. Conclui-se que:

$$
B(r, t)=-\frac{R^{2}(t)}{1-k r^{2}}
$$


O valor assumido para $k$ para um universo fechado é 1 . Para os demais valores de $k(0$ ou -1$)$, o universo estará em expansão perpétua, não havendo retorno possível. Tem-se, portanto:

Tabela 1. Geometria do universo em função da constante $k$

\begin{tabular}{c|c}
\hline Valor de $k$ & Geometria do universo \\
\hline 1 & Esférico fechado \\
\hline 0 & Plano ou chato \\
\hline-1 & Hiperbólico aberto \\
\hline
\end{tabular}

Fonte: Elaborada pelos próprios autores.

O caso de um universo esférico fechado finito em expansão está de acordo com as mais recentes descobertas a respeito do "Big Bang" e da radiação cósmica de fundo (JÁCOMO, 2017; NOVELLO, 2014), segundo as quais sua energia, em micro-ondas, atualmente correspondente à temperatura de $2,73 \mathrm{~K}$, diminuirá gradualmente com a expansão até atingir as proximidades do zero absoluto, quando então terá exaurido sua energia, cessando, finalmente, a sua expansão e, com isto, delimitando as fronteiras do universo.

O estudo da física de altas energias levou à invenção dos aceleradores de partículas, em que partículas, tais como prótons e elétrons, são acelerados até próximos da velocidade da luz por gigantescos campos magnéticos e forçados a colidirem entre si.

Sabe-se que a energia concentrada liberada pelos aceleradores, tais como o enorme colisor de hádrons, pode criar uma microbolha, a qual, especula-se, poderia dar origem a um novo universo. Para maiores informações e detalhes, sugere-se consultar as obras de Michio Kaku, renomado físico teórico e autor de inúmeros best sellers, entre eles: A Física do Futuro (KAKU, 2011) e Hiperespaço (KAKU, 2000).

Um modelo plausível para o caso de uma bolha em colapso ou crescimento é o mostrado na Fig. 1.

Figura 1. Modelo do universo finito esférico.

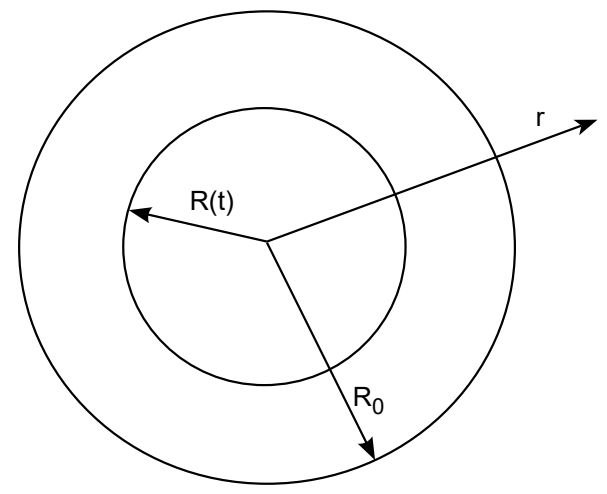

Fonte: Elaborada pelos próprios autores.

Tal modelo é similar ao de uma bolha gasosa num meio líquido, contendo ar e vapor, por exemplo. Segundo o cosmologista brasileiro Mário Novello, é possível comparar o universo a um gás contido em uma caixa que dispõe de um pistão, constituindo um sistema fechado (sem trocas de massa com o exterior). Nesse sentido, as equações da cosmologia assemelham-se bastante às equações da dinâmica de cavidades esféricas, ou bolhas, dotadas de inércia e/ou um sinal externo, como um pulso variável no tempo (NOVELLO, 2014). A geometria de um universo se expandindo desde seu início até um volume máximo e depois se contraindo é possível e utilizada em diversos modelos da dinâmica de cavidades (FRANK; MICHEL, 2005), de modo que são observadas semelhanças entre as equações da energia de uma bolha gasosa/vaporosa e as equações do universo esférico finito. Por exemplo, desprezando as tensões interfaciais, obtém-se a Eq. (4) (BAZANINI, 2003), a qual terá sua origem comentada na seção seguinte, abaixo: 


$$
\frac{d P}{d t}=-3 R^{2} R^{\prime}\left[\frac{K_{g} P_{g_{0}} R_{0}^{3 K_{g}}}{\left(R^{3}-a_{g}^{3}\right)^{1+K_{g}}}+\frac{K_{v} P_{v_{0}} R_{0}^{3 K_{v}}}{\left(R^{3}-a_{v}^{3}\right)^{1+K_{v}}}\right]-4\left(\mu_{g}+\mu_{L}\right)\left(\frac{R R^{\prime \prime}-R^{\prime 2}}{R^{2}}\right)
$$

Onde o subscrito $v$ aplica-se ao vapor, o subscrito $g$ aplica-se ao gás e o subscrito $L$ aplica-se a líquido. $P$ é a pressão, e $R$ é o raio da bolha. Os sobrescritos denotam derivação temporal. Os subscritos 0 referem-se às condições iniciais. $\mu_{L}$ e $\mu_{g}$ são as viscosidades do gás e do líquido, respectivamente. O conteúdo da bolha é tratado como fluido real através de $a_{g}$ e $a_{v}$ (BARBER et al., 1997). Além disso, $k_{v}$ e $k_{g}$ são as constantes adiabáticas do vapor e do gás.

Para efeito de comparação, a partir das equações de Einstein da relatividade geral, pode-se obter (LANDAU; LIFSHITZ, 1980):

$$
\frac{1}{8 \pi G}\left[\left(\frac{R^{\prime}}{R}\right)^{2}+2 \frac{R^{\prime \prime}}{R}+\frac{k c^{2}}{R^{2}}\right]=\frac{1}{3 R^{2} R^{\prime}} \frac{d\left(\rho R^{3}\right)}{t}
$$

Onde $G$ é a constante gravitacional e $\rho$ é a densidade.

$$
G=\frac{k c^{2}}{8 \pi}
$$

Bolhas formadas no interior de líquidos devido à cavitação (formação, crescimento e colapso de bolhas) vaporosa envolvem a fase vapor, havendo, também, em geral, a presença de ar. Como na grande maioria das situações de interesse prático todo líquido contém certa quantidade de ar dissolvido, toda bolha deve conter ar em seu interior, já que o mesmo atua como fonte de nucleação para a bolha.

O processo tem início a partir dos chamados núcleos de cavitação, os quais são constituídos por aglomerados de moléculas de ar, em número suficiente para permitir o início da formação das bolhas. Esses núcleos, ou microbolhas, possuem diâmetros da ordem de $10^{-5}$ a $10^{-3}$ centímetros. Observe-se que nunca teremos o valor zero para o raio da bolha, seja crescendo (expandindo), seja colapsando (contraindo).

O processo de crescimento e colapso das bolhas engloba a ação de forças de pressão em contraposição devido à tensão superficial, inércia e origem viscosa. Após a nucleação e o crescimento, tem-se o que poderia ser chamado de bolha formada. Quando submetida a um campo de pressões com valores superiores ao da pressão parcial do vapor mais a pressão parcial do ar, tem início o seu colapso. O colapso de bolhas é um fenômeno de curta duração (da ordem de milissegundos).

De uma maneira geral, a formação e o crescimento de bolhas ocorrem em regiões onde a pressão é suficientemente baixa para vencer a resistência imposta pela tensão superficial na interface.

Desde o trabalho de Rayleigh (1917) sobre o colapso de uma bolha esférica vazia, diversas equações têm sido propostas ao longo das últimas décadas visando explicar a formação, o colapso e o crescimento de bolhas de ar ou vapor em meios líquidos.

Alguns outros trabalhos interessantes mostrando colapso ou oscilações de bolhas podem, ainda, ser citados: Gilmore (1952), Keller e Kolodner (1956) e Prosperetti (1986), Muller et al. (2012) e Bazanini e Bressan (2017), em que as simulações são validadas mediante medições do raio da bolha em função do tempo. A formação de bolhas para estudos experimentais pode ser realizada com equipamentos destinados ao ensaio experimental, tais como o equipamento a disco rotativo (BAZANINI et al., 2017).

Nos estágios finais do colapso de bolhas ocorrem, ainda, dois fenômenos: ondas de choque e microjatos, responsáveis pela erosão em estruturas e equipamentos hidráulicos.

Existem duas possibilidades para o formato dos microjatos gerados pelo colapso das bolhas. Para a primeira, será utilizada a ferramenta matemática "escoamento potencial" (COLE, 1962), em que o escoamento 
pode ser "visualizado" através de suas funções correntes de fluxo $\psi$, já que são paralelas ao vetor velocidade em cada instante. A cada linha do escoamento corresponde um valor de $\psi$ constante. Fazendo uma analogia entre o escoamento do microjato através da cavidade (ou bolha) com o escoamento através de uma fenda, obteremos para as funções $\psi$ constantes do escoamento potencial:

$$
\frac{x^{2}}{c^{2} \cos ^{2} \psi}-\frac{y^{2}}{c^{2} \operatorname{sen}^{2} \psi}=1
$$

Onde $c$ é uma constante real (na verdade, é a distância focal da hipérbole). A utilização do escoamento potencial justifica-se devido à baixa viscosidade da água.

A Equação (7) acima representa a equação de uma hipérbole, indicando que o formato dos microjatos pode ser hiperbólico, de acordo com esse modelo.

Lembrando que as equações de uma hipérbole, na forma canônica, são (VENTURI, 2003):

$$
\frac{x^{2}}{a^{2}}-\frac{y^{2}}{b^{2}}=1
$$

E

$$
c^{2}=a^{2}+b^{2}
$$

Onde $c$ é a distância focal e a é a distância.

A excentricidade da hipérbole é definida como:

$$
\varepsilon=\frac{c}{a}
$$

Outra possibilidade seria fazer uma analogia entre o escoamento através da cavidade com o jato escoando através de um tubo estreito. Nesse caso, o jato saindo do tudo teria formato cônico, com um ângulo de 12,5 a 17,5 graus em relação à vertical (LANDAU; LIFSHITZ, 1987).

Figura 2. Microjato cônico.

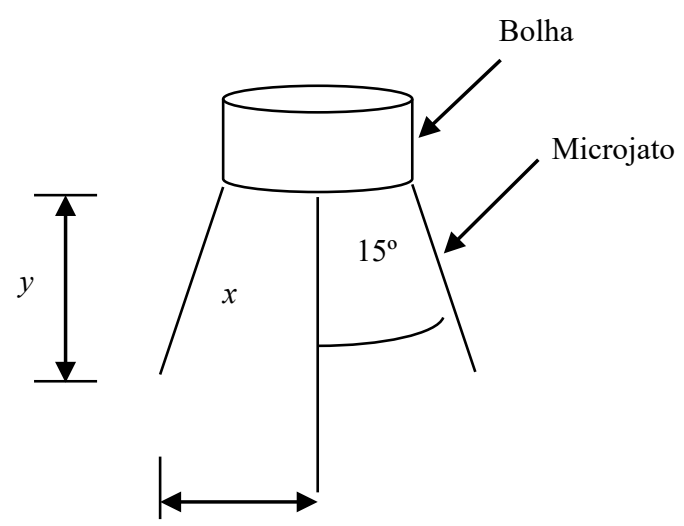

Fonte: Elaborada pelos próprios autores.

A cota $x$ da Figura 4 será, portanto, igual à metade do diâmetro do pit de cavitação $\varnothing_{\text {pit }}$ obtido experimentalmente neste trabalho, ou seja: 


$$
x=\frac{\phi_{\text {pitt }}}{2}
$$

A cota y representa a distância da bolha ao corpo de prova.

\section{Metodologia}

As simulações foram iniciadas a partir de um raio inicial máximo da bolha (correspondente ao tamanho máximo do universo finito), quando se inicia seu colapso ou contração.

Uma forma atual dessa equação, bastante completa no sentido de considerar as propriedades físicas dos fluidos, assim como a compressibilidade do líquido e a viscosidade, é a Eq. (12) abaixo. A dependência do tempo deve-se ao fato de que o raio $R$ é função do tempo, $(R=R(t))$.

Para resolver a Eq. (12), primeiro, foi necessário obter a derivada do termo $d P / d t$ no tempo (BAZANINI, 2003), que resultou na Eq. (4).

$$
\left(1-\frac{R^{\prime}}{C}\right) R R^{\prime \prime}+\frac{3}{2}\left(1-\frac{R^{\prime}}{3 C}\right) R^{\prime 2}=\frac{1}{\rho_{L}}\left[\left(1+\frac{R^{\prime}}{C}\right) P_{L}+\frac{R}{C} \frac{d P}{d t}-\left(1+\frac{R^{\prime}}{C}\right)\left(P_{\infty}-P_{A} \sin \omega t\right)\right]
$$

Onde:

$$
P=\frac{P_{g_{0}} R_{0}{ }^{3 K_{g}}}{\left(R^{3}-a_{g}^{3}\right)^{K_{g}}}+\frac{P_{v_{0}} R_{0}{ }^{3 K_{v}}}{\left(R^{3}-a_{v}^{3}\right)^{K_{v}}}-\frac{2 S}{R}-\frac{4\left(\mu_{g}+\mu_{L}\right)}{R} R^{\prime}
$$

E:

$$
\frac{d P}{d t}=-3 R^{2} R^{\prime}\left[\frac{K_{g} P_{g_{0}} R_{0}^{3 K_{g}}}{\left(R^{3}-a_{g}^{3}\right)^{1+K_{g}}}+\frac{K_{v} P_{v_{0}} R_{0}^{3 K_{v}}}{\left(R^{3}-a_{v}^{3}\right)^{1+K_{v}}}\right]-4\left(\mu_{g}+\mu_{L}\right)\left(\frac{R R^{\prime \prime}-R^{\prime 2}}{R^{2}}\right)
$$

$P_{\infty}$ é a pressão inicial externa à bolha (no meio líquido, portanto), $R_{0}$ é o raio inicial da bolha, $P_{g 0}$ é a pressão inicial do gás (ar) dentro da bolha e $\mathrm{P}_{v 0}$ é a pressão inicial do vapor. $\mu_{L}$ e $\mu_{g}$ são as viscosidades do líquido e do gás, respectivamente. $K_{v}$ e $K_{g}$ são as constantes adiabáticas do vapor e do gás. $C$ é a velocidade do som no meio líquido (água em nosso caso), $a_{g}$ e $a_{v}$ são as constantes de van der Waals ('hard core radius') para o gás e o vapor, respectivamente (BARBER et al., 1997), e $\rho_{L}$ é a densidade do líquido. É ainda considerado na Eq. (12) um termo externo de excitação da bolha na forma de um sinal variável de pressão a que o universo também pode estar sujeito (HAWKING, 2016), de amplitude $P_{A}$ e frequência $\omega$. Tal fator, em analogia com a aceleração da expansão do universo, tem sido associado, ou interpretado, como a ação da (ainda) misteriosa "energia escura" (HAWKING, 2016). Assim, a pressão inicial externa $P_{\infty}$ pode ser variada em função do tempo com a amplitude $P_{A}$ e a frequência $\omega$. Considerou- se que o gás e o vapor aprisionados na bolha obedecem à equação de estado de van der Waals para gases reais.

\section{Resultados e discussões}

Trabalhando com o sistema de equações acima, podem-se obter as oscilações formadas pelos sucessivos crescimentos e colapsos de uma bolha de ar e vapor em água para as condições iniciais e de contorno citadas. O raio adimensional é em relação ao raio inicial da bolha $\left(r=R / R_{0}\right)$, e o tempo adimensional $T$ é (MIKSIS; TING, 1984): 


$$
T=\frac{\mathrm{t}}{\mathrm{R}_{0}} \sqrt{\frac{P_{\infty}}{\rho_{L}}}
$$

O presente modelo difere dos anteriores nos seguintes aspectos: os autores consideram efeitos térmicos no gás (apenas o ar é considerado no interior da bolha; a presença de vapor é desconsiderada por eles); utilizam relação politrópica para o gás com expoente 1,4; e não consideram a compressibilidade do líquido próximo à bolha (o que ocorre nos estágios finais de cada colapso). No presente modelo, a compressibilidade do líquido é levada em consideração e o conteúdo da bolha, que é formado por ar e vapor d'água (já que a bolha cresce com a formação de vapor), é tratado como uma mistura de gases reais (BARBER et al., 1997). Embora praticamente desprezível neste caso, a viscosidade do gás também é considerada neste modelo, para que a equação fique na forma mais completa possível, uma vez que assim pode ser aplicada a quaisquer outros fluidos. Os resultados obtidos nestas simulações são mostrados nas Figs 3 a 5 .

Podem-se observar semelhanças entre as Figs. 3, 4 e 5, em que as diferenças podem ser atribuídas aos seguintes fatores: diferenças nas hipóteses utilizadas, como descrito no parágrafo anterior; diferenças no modelo aqui utilizado que, por ser mais completo em essência, alguns valores de propriedades físicas tiveram que ser estabelecidos, tais como os dados para o vapor d'água e a velocidade do som, por exemplo; além disso, o modelo proposto considera o início do primeiro colapso a partir do ponto de máximo raio inicial da bolha e a adimensionalização foi feita em relação a esse raio inicial.

Outra possibilidade de adimensionalização para o tempo é (NEPPIRAS, 1980):

$$
T \cdot=\frac{t}{R_{0}} C
$$

Essa adimensionalização foi utilizada na obtenção das Figs. 3 a 5, variando-se alguns dos parâmetros. Foram consideradas, nessas simulações, como condições iniciais e de contorno no sistema internacional de unidades: $P_{\infty}=10^{5} \mathrm{~Pa}$ (pressão atmosférica ao nível do mar); $R_{0}=0,1 \mathrm{~cm}$ ("FRANK; MICHEL, 2005); $P_{g 0}=40$ $\mathrm{Pa}$ (medida por Hammitt, 1980 ); $\mathrm{P}_{\mathrm{vo}}=2340 \mathrm{~Pa}$ (medida por Hammitt, 1980); $\mathrm{S}=0,0725 \mathrm{~N} / \mathrm{m}$ (tensão interfacial da água); $\mu_{L}=0,001 \mathrm{~kg} / \mathrm{ms} ; \mu_{g}=1,81 \times 10^{-5} \mathrm{~kg} / \mathrm{ms} ; K_{v}=1,328 ; K_{g}=1,4 ; C=1481 \mathrm{~m} / \mathrm{s}$ (LÖFSTEDT et al., 1993); $R_{d} a_{g}=8.54$ (BARBER et al., 1997); $R_{d} a_{v}=10.79$ (BARBER et al., 1997) e $\rho_{L}=998 \mathrm{~kg} / \mathrm{m}^{3}$ (densidade da água líquida).

Figura 3. Raio da bolha em função do tempo para: $P_{\infty}=10^{5} \mathrm{~Pa} ; R_{0}=$ $0,1 \mathrm{~cm} ; P_{A}=50000 \mathrm{~Pa} ; \omega=10000 \mathrm{~s}^{-1} ; \omega=10000 \mathrm{~s}^{-1}$

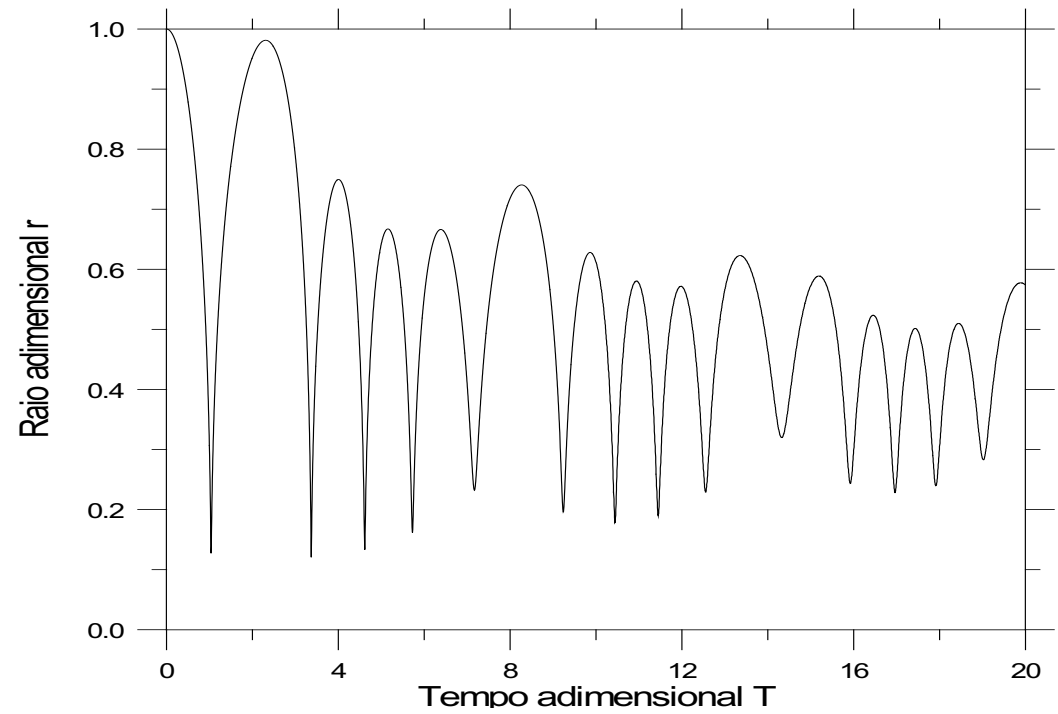

Fonte: Elaborada pelos próprios autores. 
Figura 4. Raio da bolha em função do tempo para: $P_{\infty}=10^{5}$

$$
\mathrm{Pa} ; R_{0}=2,6 \times 10 \mathrm{e}^{-5} \mathrm{~m} ; P_{\mathrm{A}}=33300 \mathrm{~Pa} ; \omega=83400 \mathrm{~Hz} \text {. }
$$

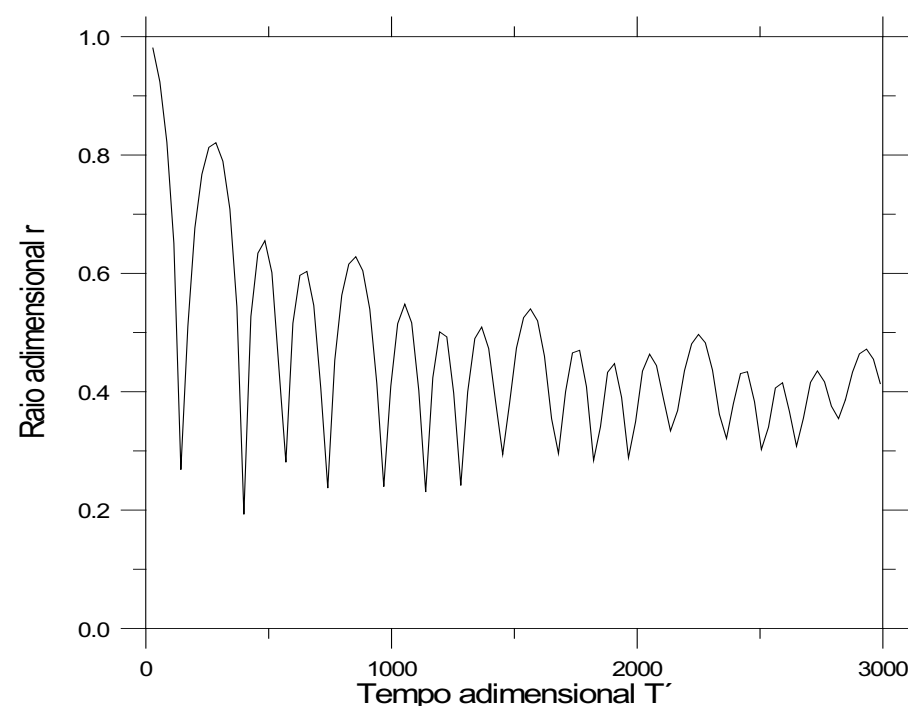

Fonte: Elaborada pelos próprios autores.

As diferenças entre as figuras obtidas com resultados do presente modelo e outros existentes são devidas à necessidade de se estabelecer valores (medidos ou calculados) às variáveis complementares do modelo atual. O modelo atual considera compressão e expansão de gases reais como conteúdo da bolha.

Os resultados obtidos nesta seção apontam para uma situação em que o raio (e, portanto, o volume) da bolha, ou cavidade (e consequentemente do universo), nunca atinge o valor zero; antes disso, inicia-se uma nova expansão, o que também está de acordo com o modelo proposto por Novello (2014) e modelos atuais do "Big Bang", nos quais o raio inicial do universo é diferente de zero. Podem-se variar os parâmetros, obtendo-se diferentes configurações semelhantes de um modelo "bouncing universe" (universo oscilante eterno), sempre sem a necessidade de se recorrer às indesejáveis singularidades presentes nos primeiros modelos: raio zero e densidade e massa infinitas. Vide também "O Universo Numa Casca de Noz" (HAWKING, 2016).

Figura 5. Raio da bolha em função do tempo para: $P_{\infty}=10^{5}$

$$
\mathrm{Pa} ; R_{0}=2,6 \times 10 \mathrm{e}^{-5} \mathrm{~m} ; P_{\mathrm{A}}=33300 \mathrm{~Pa} ; \omega=191500 \mathrm{~Hz} \text {. }
$$

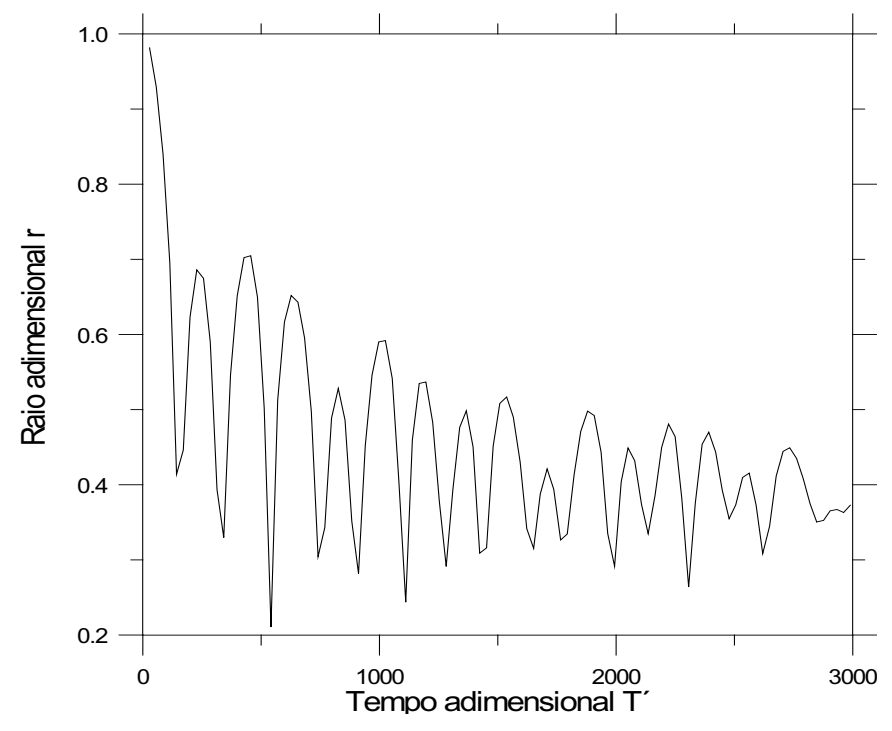

Fonte: Elaborada pelos próprios autores. 


\section{Considerações finais}

O modelo apresentado é mais completo que outros existentes para cavitação por considerar a presença de ar mais vapor como gases reais dentro da bolha, além das propriedades físicas dos fluidos participantes do processo, tais como viscosidade, densidade, tensão superficial e compressibilidade do líquido. Esse modelo mostrou-se bem-sucedido na obtenção das oscilações estáveis não lineares de uma bolha contendo ar e vapor em água líquida. Essas oscilações aparecem em gráficos de raio em função do tempo, ambos na forma adimensionalizada.

As diferenças entre os resultados obtidos com o presente modelo e os de outros autores devem-se às diferentes hipóteses estabelecidas em cada modelo, bem como à necessidade de escolha dos valores das novas variáveis que aparecem no modelo atual, tais como propriedades do vapor d'água e velocidade do som.

O modelo atual considera uma mistura de ar e vapor como gases reais. Embora o modelo proposto seja mais completo, em essência, ainda não é possível afirmar que o mesmo proporcione resultados mais precisos que os anteriores, pois seria necessário comparar com resultados experimentais nas mesmas condições das simulações aqui efetuadas.

O modelo de cavidades preenchidas com ar e vapor aqui utilizado também é condizente com modelos atuais de cosmologia, nos quais o raio do universo nunca atinge o valor zero, ou seja, não existem singularidades, tais como raio zero e densidade infinita.

Se nosso universo seguir um processo de expansões e compressões similares, o resultado será fatalmente o de um universo finito oscilante eterno, compatível com o modelo do cosmologista brasileiro Mário Novello.

\section{Referências}

BARBER, B. P. et al. Defining the unknowns of sonoluminescence. Physics Reports, USA, v. 281, n. 2, p. 65-143, mar. 1997.

BAZANINI, G. Temperature calculation inside collapsing bubbles in compressible liquids. In: BRAZILIAN CONGRESS ON MANUFACTURING ENGINEERING, 2., Uberlandia, 2003. Anais [...]. Uberlândia: Associação Brasileira de Ciências Mecânicas, 2003.

BAZANINI, G.; BARBOSA Jr., A. F.; LIMA, N. N. C. Erosion and corrosion by micro-jets and high temperature cavity impaction on metal surfaces. In: BRAZILIAN CONGRESS ON MANUFACTURING ENGINEERING, Joinville, Brazil, 2017. Anais [...]. Joinville: Associação Brasileira de Ciências Mecânicas, 2017. Disponível em: https://eventos.abcm.org.br/cobef2017/proceedings/. Acesso em: 25 abr. de 2019.

BAZANINI, G.; BRESSAN, J. D. Hot vapor bubble prints on carbon steel. Journal of Applied Mathematics and Physics, USA, v. 5, p. 439-448, 2017.

BERMAN, M. S.; GOMIDE, G. Cálculo tensorial e relatividade geral. uma introdução. São Paulo: McGraw-Hill, 1987.

COLE, G. H. A. Fluid dynamics - Methuen's monographs oh physical subjects. London: Great Britain, 1962.

EINSTEIN, A. The meaning of relativity. 4. ed. Princeton: Princeton University Press, 1953.

FRANK, J. C.; MICHEL, J. P. Fundamentals of cavitation. Dordrecht: Kluwer Academic Publishers, 2005.

GILMORE, F. R. The growth or collapse of a spherical bubble in a viscous compressible liquid. Report No. 26-4. Office of Naval Research. California Institute of Technology Hydrodynamics Laboratory, abr. 1952.

HAMMITT, F. G. Cavitation and multiphase flow phenomena. New York: McGraw-Hill, 1980. 
HAWKING, S. O universo numa casca de noz. 2. ed. Rio de Janeiro: Editora Intrínseca, 2016.

JÁCOMO, G. Universo rodopiante. Porto Alegre: Ledur Serviçoes Editoriais, 2017.

KAKU, M. A física do futuro. São Paulo: Rocco, 2011.

KAKU, M. Hiperespaço. São Paulo: Rocco, 2000.

KELLER, J. B.; KOLODNER, I. I. Damping of underwater explosion bubble oscillations. Journal of Applied Physics, USA, v. 27, n. 10, p. 1152-1161, out. 1956.

LANDAU, L. D.; LIFSHITZ, E. M. Teoria do campo. São Paulo: Hemus, 1980.

LANDAU, L. D.; LIFSHITZ, E. M. Fluid mechanics. 2. ed. Oxford: Pergamon Press, 1987.

LÖFSTEDT, R.; BARBER, B. P; PUTTERMAN S. J. Toward a hydrodynamic theory of sonoluminescenc. Physics of Fluids A, USA, v. 5, n. 11, p. 2911-2928, nov. 1993.

MIKSIS, M. J.; TING, L. Nonlinear radial oscillations of a gas bubble including thermal effects. Journal of the Acoustical Society of America, USA, v. 76, n. 3, p. 897-905, set. 1984.

MULLER, M. A. S.; ZIMA, P.; UNGER, J. Energy dissipated during the cavitation bubble collapse close to a solid wall. In: INTERNATIONAL SYMPOSIUM ON CAVITATION, 8., Singapore, 2012. Proceedings [...]. Singapore: Research Publishing Services, 2012.

NEPPIRAS, E. A. Acoustic cavitation. Physics Reports, Holland, v. 61, n. 3, p. 159-251, 1980.

NOVELLO, M. Do big bang ao universo eterno. Rio de Janeiro: Zahar, 2014.

PROSPERETTI, A. Bubble phenomena in sound fields. Part Two. Ultrasonics, England, Vol. 41, Nro. 624, p. 115-124, May, 1986.

RAYLEIGH, J. W. S. August, on the pressure developed in a liquid during the collapse of a spherical cavity. Philosophical Magazine, England, v. 34. n. 200. p. 94-98, 1917.

RIVELLES, V. O. Introdução à gravitação e à cosmologia. Escola Norte-Nordeste de Partículas e Campos. João Pessoa, 10-14/08/2009. Disponível em: http://itec.if.usp.br/ rivelles/Seminars/cosmologia_ joao_pessoa1.pdf Acesso em: 20 abr. de 2019.

VENTURI, J. J. Cônicas e quádricas. 5. ed. Curitiba: Artes Gráficas e Editora Unificado, 2003. 
Sobre os autores

\section{Gil Bazanini}

Doutorado em Engenharia Mecânica pela Universidade Federal da Paraíba (1998) e mestrado em Engenharia Mecânica pela Universidade de São Paulo (1992). Possui graduação em Engenharia Mecânica pelo Centro Universitário da FEI (1986). Atualmente, é professor adjunto da Universidade do Estado de Santa Catarina, atuando principalmente nos seguintes temas: cavitation, bubbles, cavity e fibonacci.

\section{Ricardo Kirchhof Unfer}

Doutorado em Ciência e Engenharia de Materiais pela Universidade do Estado de Santa Catarina (UDESC - 2015), mestrado em Ciência e Engenharia de Materiais pela Universidade do Estado de Santa Catarina (UDESC - 2004) e graduação em Engenharia Mecânica pela Universidade do Estado de Santa Catarina (UDESC - 1999). Professor colaborador da Universidade do Estado de Santa Catarina - UDESC, tem experiência na área de Engenharia Mecânica, atuando principalmente nos seguintes temas: ensaio de tração, ondulação da rugosidade (waviness), conformação de chapas, avaliação de parâmetros de rugosidade (roughness parameters) e estiramento biaxial (biaxial stretching).

\section{Nicodemus Neto da Costa Lima}

Mestrado em Engenharia Mecânica pela Universidade Federal de Santa Catarina (1985). Possui graduação em Engenharia Mecânica pela Universidade Federal de Pernambuco (1978). Atualmente, é professor da Universidade do Estado de Santa Catarina. Tem experiência na área de Engenharia Mecânica, com ênfase em mecânica dos sólidos.

Recebido em: 29/08/2019

Aceito em: 12/12/2019 